\title{
Come diventare una donna di successo nel periodo Kamakura (1185-1333). Consigli utili dalla Lettera di Abutsu
}

\author{
Carolina Negri \\ Università Ca' Foscari di Venezia \\ carolina.negri@unive.it
}

\begin{abstract}
The paper focuses on Abutsu's Letter, the first career guide for younger noble-women written by Nun Abutsu (1225-1283). This work is a long letter to her only daughter, Ki no Naishi, to provide good advice on some important matters such as proper deportment, necessary artistic skills and the right path to succeed in life at court. By analising Abutsu's Letter the paper tries to reconstruct the daily life of medieval noble women, their upbringing and aspirations. It also casts light on women's innermost feelings and fears in an era of great changings, exploring what kind of practical solutions they have if their dreams go unfulfilled.
\end{abstract}

\section{Keywords}

Nun Abutsu - medieval Japan - women's upbringing - ladies in waiting - life at court - nunhood

\section{La monaca Abutsu: una donna intraprendente del medioevo giapponese}

Scarse sono le notizie tramandate dalle fonti sulla vita della monaca Abutsu, una poetessa di grande talento che, grazie alla sua fama e ai legami del padre ${ }^{1}$ con la corte, entrò molto presto a far parte dell'entourage della principessa

1 Taira no Norishige, ufficiale dell'esercito imperiale, che potrebbe essere il padre naturale o adottivo di Abutsu. 
Ankamonin (1209-1283). Come per altre donne letterate nell'antico Giappone, non conosciamo neanche il suo vero nome. A corte, nell'ultimo periodo in cui prestò servizio, era conosciuta con il nome Ankamonin shijō, mentre Abutsu è l'appellativo con il quale viene designata solo a partire da un certo punto della sua vita quando, dopo il matrimonio con Fujiwara no Tameie (1198-1275), discendente di un'illustre famiglia di poeti, decise di prendere i voti. Con questo ultimo nome è citata solitamente negli studi critici e nelle biografie a lei dedicati, dove spesso è riportata l'impossibilità di risalire con esattezza alle date di nascita e di morte: in base ad alcune informazioni presenti nei suoi componimenti poetici, esse dovrebbero collocarsi tra il $1225 \mathrm{e} \mathrm{il} 1283 .{ }^{2}$

Proprio le vaghe coordinate temporali e le insoddisfacenti notizie biografiche di cui disponiamo ci spingono a indagare di più sulla sua ricca produzione letteraria che comprende diari, poesie, manuali, saggi critici e preghiere, utili a ricostruire con maggiore precisione il ritratto di una donna medievale molto ambiziosa che ha vissuto una vita per certi aspetti privilegiata, ma allo stesso tempo pure costellata di numerose sofferenze affrontate sempre con grande determinazione. Attraverso la sua esperienza di dama, madre, monaca, studiosa, insegnante, moglie e vedova, Abutsu riassume il destino di molte donne spesso ingiustamente ignorate nonostante la loro attiva partecipazione alla vita culturale e sociale. La mancanza di interesse nei confronti delle opere scritte dalle donne in epoca Kamakura, spesso considerate una mera imitazione di generi di epoche precedenti (ci riferiamo soprattutto ai nikki, ai setsuwa e ai waka), sembrerebbe dovuta al persistere di una scarsa integrazione tra le ricerche letterarie e storico-sociali che solo in tempi recenti, grazie al contributo di alcuni studiosi (ad es. Laffin 2013), hanno iniziato a procedere parallelamente restituendo ad alcune opere il loro meritato valore e soprattutto la funzione di privilegiato mezzo espressivo della condizione femminile in un'epoca di grande trasformazione.

Tra i cambiamenti più importanti a cui si assiste in Giappone nell'epoca Kamakura va segnalato innanzitutto il declino della corte e la necessità per le donne di contare sulle finanze di padri e mariti. In una società in cui si affermarono modelli patriarcali di stampo confuciano anche il matrimonio cambiò rispetto alle epoche precedenti. La sposa non viveva più in una residenza preparata dalla famiglia d'origine, ma si trasferiva in quella del marito al quale consegnava i suoi beni con la speranza di mettere al mondo almeno un figlio. La perdita dell'indipendenza economica e l'impossibilità per le donne di ereditare erano una naturale conseguenza dell'evoluzione del governo militare, che considerava cruciale il controllo della terra, una proprietà fissa che non

2 Sulla vita di Abutsu si veda Nagasaki, Hamanaka (1996) e Tabuchi (2009b). 
doveva essere divisa o frammentata quando i figli si sposavano. Da qui derivava per gli uomini la necessità di accoppiarsi con donne di rango più basso, spesso nominalmente acquisite nella famiglia del marito guerriero come semplici serve (Wakita 1984: 87-88). Nel caso di una donna ben istruita e di nobili origini l'alternativa al matrimonio con un uomo comune poteva essere ancora la corte dove una dama al servizio di un'imperatrice avrebbe potuto continuare senza difficoltà la sua formazione. Con un po' di fortuna poteva poi anche sposare un principe o un imperatore dal quale avere dei figli contribuendo così a innalzare il livello sociale della famiglia di origine. La nuova società non lasciava molta scelta alle donne: o si adeguavano alle regole di una rigida organizzazione patriarcale o tentavano strade alternative che richiedevano sempre molto coraggio e intraprendenza. Esemplare da questo punto di vista è la vita di Abutsu che, secondo Fukuda Hideichi (1969: 402), "a differenza di altre donne dell'epoca Heian, non si è abbandonata passivamente al suo destino, rappresentando un esempio di donna attiva del medioevo che nel bene e nel male è stata artefice della sua sorte". La necessità di essere una "donna attiva" dell'epoca Kamakura (kōdōtekina Kamakura josei nelle parole di Fukuda) che lotta con determinazione per conquistare il suo posto nel mondo è alla base della compilazione di Abutsu no fumi ("Lettera di Abutsu", 1264) una sorta di manuale per la formazione di una futura dama di corte che l'autrice dedica alla figlia quattordicenne Ki no Naishi (1251-?), all'epoca già da qualche anno al servizio di Higashi Nijō (Saionji Kōshi, 1232-1304), consorte dell'imperatore GoFukakusa (1243-1304). La lettera presenta in modo chiaro le qualità che deve avere una dama di corte e quali sono le strategie utili per conquistare il supporto di un uomo molto influente. Può essere letta allo stesso tempo sia come una riflessione sulla vita personale di Abutsu, soprattutto in riferimento alla sua esperienza di dama, sia come un chiaro iter educativo tracciato di proposito per la figlia perché seguendolo potesse raggiungere senza problemi il successo nella vita realizzando così i sogni della madre che l'aveva allevata da sola con grandi sacrifici.

\section{Lettera della nutrice o Lettera di Abutsu?}

Il testo di Abutsu no fumi ha avuto una larga diffusione per un lungo periodo ed è conosciuto pure come Menoto no fumi ("Lettera della nutrice") e Niwa no oshie ("Precetti della casa"). Le versioni del testo che sono circolate nel corso degli anni possono essere divise in due grandi categorie: una lettera più lunga, che presumibilmente Abutsu scrisse alla figlia, e una versione ridotta divenuta poi manuale di riferimento per le dame e più tardi anche per tutte le altre 
donne. Non si è ancora riusciti a stabilire con assoluta certezza se la forma originale consista nella versione "ampliata" (kōhon) o nella versione "ridotta" (ryakuhon). Le teorie degli studiosi al riguardo sono molto diverse e se non manca chi tende a riconoscere entrambe come opere della stessa autrice, non sono pochi neanche quelli che non le attribuiscono nessuna delle due. Matsumoto Yasushi (1983: 152) ritiene che entrambe siano state scritte da Abutsu, probabilmente negli ultimi anni della sua vita, mentre si trovava a Kamakura, riconoscendo a quest'opera un grande valore non solo perché fornisce molte informazioni sulla vita, la cultura e il modo di pensare dell'autrice, ma anche perché ricorda da vicino l'esperienza di altre dame della stessa epoca e come il Towazugatari ("La storia della Dama Nijō", XIV sec.) risulta un testo indispensabile per comprendere l'essenza della letteratura femminile della corte.

Una posizione diversa rispetto a quella di Matsumoto è rappresentata invece da Iwasa Miyoko (1999: 90) che ha avanzato l'ipotesi di una versione 'ampliata', scritta da Abutsu per la figlia tra il 1263 e il 1264, e di una versione 'ridotta', realizzata con molta probabilità successivamente da un'altra persona, che avrebbe variato la disposizione degli argomenti trattati nel testo originale rendendo il contenuto più frammentario e indebolendo il tema centrale dell'educazione di una dama. Sempre la stessa Iwasa, che con le sue teorie ha dato un importante impulso allo sviluppo delle ricerche sull'opera di Abutsu, basandosi su quanto riportato nel Gunshoruijü ${ }^{3}$ ("Raccolta di testi di riferimento") sostiene sia giusto indicare con Menoto no fumi la versione 'ampliata' del testo, mentre quella 'ridotta' sarebbe invece opportuno chiamarla Niwa no oshie. Anche riguardo alla denominazione del testo non c'è però parere unanime tra gli studiosi e se Iwasa distingue nettamente i titoli delle due versioni, Tabuchi Kumiko ha negli ultimi anni puntualizzato che Abutsu no fumi è scritto sia sul testo più antico della versione 'ampliata', conservato nella collezione Yōmei Bunko a Kyōto, sia su quello più lontano della versione 'ridotta', presente nella biblioteca del Kokubungaku kenkyū shiryōkan a Tōkyō, arrivando così alla conclusione che sia più giusto chiamare entrambe con questo stesso nome. Sempre secondo Tabuchi è inoltre probabile che, trattandosi di una lettera privata, in origine non vi fosse neanche un vero e proprio titolo. Considerato il contenuto e il rapporto dell'autrice con la destinataria, non sembrerebbe infatti molto adeguato il titolo Menoto no fumi probabilmente modellato su altri testi di istruzione femminile (jokun) redatti in epoca Muromachi (1333-1568), come ad esempio il racconto umoristico Menoto no sōshi ("Il libretto della nutrice") che parla di

3 Si tratta di una raccolta di documenti letterari e storici giapponesi compilata da Hanawa Hakiichi (1746-1821) nel 1779 . 
due fanciulle allevate da due diverse nutrici con principi educativi del tutto differenti che condizionano irrimediabilmente il loro futuro (Tabuchi 2009a: 96).

Nonostante la larga circolazione che ha avuto quella che d'ora in avanti, seguendo le indicazioni di Tabuchi, chiameremo Lettera di Abutsu, non esistono a oggi edizioni critiche che renderebbero più agevole la lettura del testo se non una risalente agli anni ' 30 conservata nel Kokkai toshokan a Tōkyō e definita in copertina jokun, ovvero manuale di istruzione femminile. Molto interessante è la prefazione dalla quale si evince che l'opera, al pari di altri classici, è stata riscoperta nell'epoca Shōwa (1926-1989) come testo educativo femminile perché si riteneva che, in seguito alla forte influenza della cultura occidentale iniziata nell'epoca Meiji (1868-1912), "non erano pochi i casi in cui si assisteva al declino delle qualità peculiari delle donne giapponesi" e in particolare "alla progressiva scomparsa di affabilità, grazia, semplicità e raffinatezza" (Suzuki 1936: 2). La lettura dell'opera di Abutsu dovrebbe dunque avere l'importante scopo di far riscoprire queste qualità della donna giapponese preservandole contro la minaccia di una eccessiva occidentalizzazione dei costumi.

\section{La destinataria della lettera}

In Abutsu no fumi l'autrice parla della nascita della figlia e con rammarico ricorda la solitudine e le difficoltà economiche del periodo in cui aveva dovuto crescerla da sola.

Tra le altre cose, ricordo con dispiacere che ti ho allevata senza avere neanche una coperta in cui avvolgerti nel periodo in cui non disponevo più dei mezzi necessari a vivere perché mi ero allontanata dai parenti $\mathrm{e}$ non potevo a maggior ragione chiedere nulla neanche alle persone più estranee [...]. Avrei voluto coprirti di vesti di broccato con fantasie di fiori primaverili o con foglie autunnali della Principessa del Tatsuta e provavo una gran pena a vederti dormire con una sola veste addosso. Nelle notti fredde scaldavo il tuo giaciglio e mi stendevo accanto a te sapendo che l'unica luce su cui potevo contare era quella della neve riflessa sulle pareti. Notte dopo notte pregavo le divinità invisibili e mi rammaricavo delle conseguenze negative della mia vita passata. Vissi così per circa due anni $[\ldots]$.

YANASE 1984: 135 
Non sappiamo con certezza perché Abutsu fosse stata costretta a vivere in condizioni di grave indigenza, ma possiamo supporre che fosse la conseguenza della sua ribellione alla volontà del padre che aveva individuato come suo futuro sposo un uomo che lei aveva rifiutato. Si era innamorata con molta probabilità di un altro, e nel corso o dopo la fine di questa relazione osteggiata dalla famiglia decise di prendere i voti e di ritirarsi nel tempio Hokkeji dal quale poi si allontanerà per dare alla luce una bambina. Proprio in questo periodo, a quanto pare, non poté più fare affidamento sui parenti e fu costretta a occuparsi da sola della figlia per almeno due anni. Da questa situazione di solitudine e precarietà fu però inaspettatamente salvata da una monaca dell'Hokkeji, che essendo venuta a sapere che GoSagain no Dainagon no Suke (1233-1263), la figlia di Fujiwara no Tameie, stava cercando per il padre una persona adatta a ricopiare il testo del Genji monogatari ("La storia di Genji", inizio XI sec.), le aveva proposto di ingaggiare Abutsu per questo scopo. Proprio l'oppotunità di svolgere un lavoro come copista si rivelò molto fortunata perché le permise, circa due anni dopo la nascita della figlia, avvenuta intorno al 1251, di conoscere Fujiwara no Tameie con il quale visse una travolgente passione destinata a cambiare radicalmente la sua vita e quella della figlia che, a quanto pare, proprio grazie alle conoscenze di Tameie, fu introdotta molto presto a corte.

Leggendo la lettera apprendiamo che a soli sette anni aveva già suonato il sō no koto 4 "al cospetto dell'imperatore abdicadario" (In no gomae). Dovrebbe trattarsi all'incirca del 1257 quando era salito al trono GoFukakusa mentre l'imperatore abdicadario era GoSagain (1220-1272). L'anno successivo, a otto anni, partecipò poi anche a un concerto suonando ancora una volta il sō. Più o meno cinque anni dopo, quando doveva avere circa tredici anni, Abutsu si allontanò dalla capitale per seguire Tameie, che intanto aveva sposato, separandosi dalla figlia alla quale decise di scrivere la lettera per farle sentire anche a distanza affetto e protezione. Alla fine della lunga epistola a lei indirizzata leggiamo: "inviata a Sua Eccellenza Ki no Naishi" (Ki no Naishi dono he mairasesorō), e se possiamo fare affidamento sull'attendibilità del titolo attribuito alla destinataria, dobbiamo dedurre che la figlia di Abutsu a tredici anni era già diventata naishi (Tabuchi 2009b: 46-50), aveva cioè raggiunto un rango molto alto a corte essendo la dama che assisteva più da vicino l'imperatore trasmettendo i suoi ordini e guidando le dame di rango inferiore. Nonostante i sacrifici fatti nei primi anni per allevarla, Abutsu non aveva mai ceduto al pessimismo e aveva continuato a nutrire grandi speranze sul futuro della figlia, riguardo al quale dichiara di avere ricevuto più volte precise visioni in sogno.

4 Tipo di cetra a tavola a tredici corde suonata con l'ausilio di unghie d'avorio. 
Prima che tu nascessi ho fatto un sogno incredibile nel quale confidavo molto: mostrava chiaramente che avrei dato alla luce una figlia femmina che avrebbe conquistato una posizione sociale molto elevata illuminando il mondo con la sua luce. Faceva poi vedere anche molti altri particolari e persino che ci sarebbero state delle persone un po' invidiose che avrebbero rivaleggiato con lei. Non riuscivo a capire perché, ma non c'erano dubbi sul fatto che saresti diventata una persona importante. Anche dopo la tua nascita, ho continuato più volte a fare lo stesso sogno. Se ci si affida alla dea Kasuga, non si può dubitare che non dica la verità.

Ricordo ancora che dentro di me aspettavo con straordinaria trepidazione che tu crescessi. Dicevo a me stessa che, fino a quando non si sarebbe realizzato quel sogno, non avrei detto niente a nessuno e lo avrei custodito nel profondo del mio cuore. La mattina appena sveglia e la sera prima di andare a dormire ho continuato a pregare diligentemente le divinità perché si avverasse. Solo adesso lo rivelo per la prima volta e penso di non farlo mai in presenza di altre persone. Le visioni che ho avuto erano senz'altro il risultato del karma di una mia precedente esistenza.

YANASE 1984: 125-26

Come riportato più avanti, Abutsu spera che la figlia riceva l'amore dell'Imperatore e che possa soprattutto dargli un figlio. Diverse fonti tra cui L'Izayoi nikki ("Diario della luna della sedicesima notte", 1280), scritto dalla stessa Abutsu, testimoniano che questo suo grande desiderio col tempo si realizzerà. Ki no Naishi ebbe infatti una figlia dall'imperatore GoFukakusa e per la sua posizione a corte, ma anche perché era una persona affidabile e matura, rappresenterà un punto di riferimento molto importante per la madre quando questa, negli ultimi anni della sua vita, deciderà di intraprendere un viaggio verso Kamakura per rivendicare davanti al tribunale del governo militare i diritti di suo figlio a ereditare dopo la morte del marito (Tabuchi $20096: 51^{-52}$ ).

\section{Formare una dama dal comportamento impeccabile}

Il contenuto della lettera può essere suddiviso in tre parti principali che rispettivamente comprendono: 1) consigli sul comportamento di una dama; 2) elementi fondamentali della sua cultura; 3) strategie per conquistare un uomo di alto rango.

I consigli relativi al comportamento di una dama aprono la prima parte dell'opera, forse non a caso, come se rappresentassero la base fondamentale, imprescindibile di un progetto educativo molto mirato. Dopo aver manifestato 
il rammarico per la separazione e allo stesso tempo il dispiacere per essere costretta a vivere lontano dalla capitale, Abutsu, commossa per aver ricevuto una lettera dalla figlia che le chiede qualche consiglio, comincia il suo lungo discorso insistendo subito sulla necessità di comportarsi sempre in modo appropriato a una donna di rango.

Devi sapere che anche la persona più bella e raffinata di questo mondo se non ha una adeguata maturità non raggiungerà mai nessuno scopo nella vita.

Quando si desidera qualcosa che sta particolarmente a cuore, per evitare che le persone possano ascoltare inavvertitamente e riferirlo anche agli altri, bisogna tenerlo solo per sé. A volte si realizza proprio quello che si vuole, ma possono pure verificarsi eventi spiacevoli uno dopo l'altro. Se capita qualcosa di molto grave, poiché è imbarazzante darlo a vedere agli altri, è meglio far finta di niente e parlare poco anche se si è davvero addolorati. Inoltre, anche quando si verifica quello che volevamo, non si deve mai dire: "Che bello! Come sono felice!". Sia le cose positive che quelle negative bisogna tenerle nascoste nel nostro cuore riflettendo su di esse attentamente. E poi bisogna considerare sempre le altre persone. Non si deve mai giudicarle dicendo: "E fatta così e così". Meglio tenere certe osservazioni solo per se e nella maggioranza dei casi, quando si tratta degli altri ma anche di noi stessi, evitare di parlare e di lasciare trapelare i nostri sentimenti con persone poco affidabili.

YANASE 1984: 109-10

In questa prima parte della lettera ritorna come un persuasivo ritornello l'espressione onkokoro no uchi nite ("nascondere i sentimenti nel cuore") che fa evidentemente riferimento a una virtù molto importante, la riservatezza, fondamentale per una dama di corte che deve imparare a reprimere le sue naturali reazioni emotive mostrandosi in apparenza sempre calma e serena (Laffin 2013: 24-25). Essendo Abutsu profonda conoscitrice di testi di ispirazione buddhista, riguardo al tema della 'riservatezza delle emozioni', non si può escludere anche una possibile influenza del Jikkunshō ("Trattato dei dieci insegnamenti", $1250 \mathrm{ca}$.), una raccolta di aneddoti scritti in un'epoca vicina a quella a cui dovrebbe risalire la Lettera, in cui si parla dellimportanza di non esternare i propri sentimenti e di quanto sia deplorevole parlare male delle altre persone (Takagi 2010: 33-34).

Nella parte iniziale della Lettera colpisce subito l'alta concentrazione della parola kokoro che da sola può significare cuore, sentimento o animo, qui ricorrente in espressioni come kokorosadamarazu ("fino a che non si raggiunge 
l'equilibrio, la maturità"), kokoro o soete ("fare attenzione"), kokoro ni kokoro o kataraite ("parlare dei sentimenti al proprio cuore") e kokoro no mama ("come si desidera"). Si può dire che proprio in queste poche espressioni è racchiusa l'essenza dell'intera lettera il cui obiettivo principale è la formazione di una giovane donna che deve imparare a relazionarsi con l'ambiente della corte reprimendo spesso le reazioni spontanee e facendo sempre attenzione al giudizio degli altri. Il raggiungimento della maturità e dell'equilibrio che contraddistinguono una persona adulta è chiaramente in netto contrasto con l'assecondare la propria volontà, tipico di una persona incauta perché giovane e inesperta.

La reputazione di una dama è senza dubbio molto importante per la sua scalata sociale perciò da buona madre Abutsu mette in guardia la figlia dagli inevitabili, pericolosi pettegolezzi che circolano a corte consigliandole di fare attenzione a non lasciarsi coinvolgere da quello che dicono gli altri.

Se chi ti sta vicino detesta una persona e si mette a spettegolare improvvisamente con te rivelandoti qualcosa di segreto, non mostrarti interessata alla conversazione. E se per caso qualcuno poi ti chiede: "Ma cosa ha detto quella persona? Di che si tratta?", rispondi con indifferenza un po' infastidita: "Mah, chissà di che si tratta! Non ho fatto attenzione a quello che diceva".

YANASE 1984: 111-12

Pur comportandosi sempre con riservatezza e il dovuto distacco quando le circostanze lo richiedono, la dama ideale descritta da Abutsu è una donna sensibile nei confronti degli altri e incline a stabilire un rapporto di confidenza con le persone più fidate (Yanase 1984: 112). Organizza tutto con il dovuto anticipo ed insegna alle altre dame a fare altrettanto (ibid.: 114). Si muove con grazia e lentezza evitando di agitarsi, come se ci fosse sempre tutto il tempo necessario per fare le cose con calma, ma è allo stesso tempo pure affidabile e capace di portare a termine i compiti che le sono stati affidati senza rimandarli più del necessario (ibid.: 111). Cura molto il suo modo di apparire in pubblico che, contrariamente a quanto si possa immaginare, non riguarda tanto il suo aspetto fisico o il suo abbigliamento, ma soprattutto la postura, i gesti, le espressioni del volto, il modo di sistemare le vesti e di rivolgersi agli altri.

Inoltre, per quanto riguarda l'aspetto fisico e il modo di comportarsi, ricordati che, anche se ci sono doti naturali che una persona può avere sin dalla nascita, migliorano molto in base all'attenzione che ci dedichi. Se ti muovi sempre in modo da non far apparire brusco neanche il più piccolo gesto che si vede da dietro, tutto sembrerà perfetto. Inutile dire 
poi che non bisogna alzare i gomiti né tanto meno muoverli di continuo. In ogni circostanza, quando incontri una persona, abbi cura di sistemarti con la dovuta calma di fronte a lei assumendo sempre un'espressione serena e rilassata. L'aspetto che avrai quando starai seduta dovrà risultare armonioso come quello di un uccello che affiora delicatamente sulla superfice dell'acqua. Stai pure attenta a non dimenticare di aggiustare bene le maniche delle vesti. Comportati in modo raffinato anche quando incontri qualcuno separata da un paravento e se pure lasci intravedere una chioma fluente di bellissimi capelli, per quanto ci possano essere buone ragioni per farlo, cerca di mostrarti timida e schiva col tuo interlocutore perché non sembri fatto di proposito.

YANASE 1984: 115-16

Per Abutsu la bellezza consiste, a quanto pare, nell'immagine più o meno gradevole della propria persona che si riesce a trasmettere agli altri. L'efficacia dell'effetto finale si raggiunge non solo grazie alle doti naturali, ma anche attraverso l'impegno e la pratica costante che richiedono molta più attenzione della cura che si può riservare al proprio aspetto fisico. Nella lettera, forse non a caso, non si parla di trucco, di acconciature dei capelli, né della scelta di capi di abbigliamento, mentre una breve sezione è dedicata solo alla scelta sapiente di una fragranza insolita, preparata personalmente con una miscela segreta per aggiungere quel pizzico di fascino e mistero utile ad attirare l'attenzione.

Ricordati che preparare l'incenso non è tanto una questione di ingredienti. Bisogna fare in modo che abbia un profumo penetrante e particolare che induce le persone a pensare com'è il tuo carattere. Non accontentarti di metterlo in gran quantità velocemente, ma preoccupati sempre di profumare con calma le tue vesti con una fragranza invitante. Se qualcuno ti chiede l'incenso, rispondi: "Non ho a portata di mano gli ingredienti", oppure: "Non sono sempre in grado di prepararlo" e fai in modo che anche gli altri lo vengano a sapere. Assicurati che dicano: "Quella persona ha un profumo davvero eccezionale", perché bisogna sempre distinguersi in tutte le cose.

YANASE 1984: 113-14

\section{Requisiti fondamentali della preparazione culturale}

La lettera contiene numerosi consigli sulla preparazione culturale di una futura dama che aspira a diventare consorte imperiale. Innanzitutto ricorda 
che bisogna sviluppare sin da giovani una adeguata sensibilità poetica che deriva dalla contemplazione diretta della natura circostante.

Anche le cose che sono fatte per essere contemplate come la luna e i fiori non sempre attirano la nostra attenzione. Ma non per questo non si devono osservare. Perché possono comunicarci sensazioni diverse a seconda del nostro stato d'animo. Sia da giovane che da persona anziana avrai le tue preferenze, e se non trovi proprio nulla che ti piace intorno a te, prova a fare qualche incursione nel lontano passato. E qualora fosse poi difficile seguire le vestigia di Hitomaro o di Akahito, o avere la stessa ispirazione che ha avuto Murasaki Shikibu, contemplando i raggi della luna riflessa sulle onde del lago Biwa prima di scrivere una storia che termina con l'incontro di Ukifune con il Maestro della Legge, invece di isolarti, perché non ti senti motivata a parlare, medita sulla luce della luna e sul profumo dei fiori e fai i preparativi necessari per recitare una poesia.

YANASE 1984: 117

Non basta però saper solo comporre una poesia. Molto importante è la calligrafia di una dama che deve dimostrare di saper utilizzare i caratteri giusti a seconda delle circostanze.

Fai attenzione a scrivere sempre con una bella grafia. Le persone hanno gusti diversi riguardo alla scrittura, e poiché dipende dalle circostanze, credo sia difficile stabilire a priori come deve essere. L'essenza di una donna si vede dalla sua grafia e anche una nota scritta senza un motivo particolare rivela il carattere di una persona e la sua disposizione d'animo. Tieni sempre la carta con l'occorrente per scrivere nella scatola sullo scaffale e scrivi con una grafia adeguata. La scrittura cinese non dovrebbe di norma piacere alle donne, però sarebbe assurdo non conoscerla almeno un po' per scriverne i caratteri e utilizzarli per i titoli delle poesie.

YANASE 1984: 118-19

Meno indispensabile della scrittura e della capacità di comporre versi a seconda delle circostanze sembra invece essere il disegno di cui basta avere una conoscenza molto limitata.

Inoltre, per quanto riguarda il disegno, anche se non acquisisci di proposito una grande competenza, esercitati in modo da saper disegnare 
le persone e ricopiare le illustrazioni delle parti più interessanti dei monogatari.

YANASE 1984: 119

Molto più importante è invece l'educazione musicale che deve iniziare molto presto. La figlia di Abutsu, secondo quanto ci viene riferito, aveva iniziato a studiare il sō no koto a soli cinque anni dimostrando subito una notevole attitudine per quello strumento. A sette anni, grazie alla sua bravura, aveva suonato al cospetto dell'imperatore abdicadario GoSagain e a otto si era esibita insieme al principe Haru no Miya ${ }^{5}$ che suonava il biwa. ${ }^{6}$ La madre si mostra orgogliosa del precoce talento musicale, ma si preoccupa comunque di raccomandarle di non tralasciare mai l'esercizio per mantenere sempre alta la sua reputazione di musicista.

Anche se sono soddisfatta della competenza che hai acquisito riguardo agli strumenti a corde come il koto e il biwa, ti raccomando di non considerare la musica un semplice passatempo e di impegnarti a raggiungere il livello più profondo di conoscenza di quest'arte.

YANASE 1984: 119

Fino a questo punto possiamo dire che i capisaldi dell'educazione femminile indicati da Abutsu coincidano con quelli presenti in alcune opere dell'epoca Heian (794-1185). Pensiamo ad esempio ai monogatari e ai nikki scritti per le donne, dove le protagoniste si distinguono per il loro straordinario talento poetico o musicale che spesso è determinante per esercitare fascino sull'altro sesso e decidere il corso del loro destino. Nelle parole di Abutsu sembrano riecheggiare i consigli di Sei Shōnagon (966-1025) che in un celebre passo del Makura no sōshi ("Appunti del guanciale", inizio XI sec.) riporta gli insegnamenti di Fujiwara no Morotada (920-969) alla figlia Hōshi.

Innanzitutto devi apprendere l'arte della calligrafia. Poi devi imparare a suonare il koto a sette corde meglio di chiunque altro. Inoltre, devi imparare a memoria tutte le poesie dei venti libri del Kokinwakashū.

ISHIDA 1996: 35

5 Si tratta del futuro imperatore Kameyama (1249-1305).

6 Liuto a quattro corde dotato di cassa armonica arrotondata e suonato con l'ausilio di un plettro. 
Impossibile non ricordare poi l'importanza del talento poetico sottolineata in testi come l'Izumi Shikibu nikki ("Diario di Izumi Shikibu", XI sec.) dove la protagonista, rinomata poetessa, riesce a conquistare grazie ai suoi versi il cuore di un principe imperiale diventando così un valido modello educativo per tutte le fanciulle di buona famiglia che ambivano a una scalata sociale (tr. it. Negri 2008). Per non parlare poi della funzione determinante che ha il suono del koto di Himegimi nel Sumiyoshi monogatari ("La Principessa di Sumiyoshi", $\mathrm{x}$ sec.), dove la musica diventa il segnale della presenza inequivocabile della bella e sfortunata protagonista che sarà, proprio grazie al suono del koto, scoperta per la prima volta e poi infine anche ritrovata dal suo innamorato (tr. it. Negri 2000). Il talento poetico e musicale, come sappiamo, sono caratteristiche distintive anche delle eroine del Genji monogatari considerato da Abutsu un manuale di riferimento imprescindibile per l'educazione femminile che poteva cambiare radicalmente il destino di una donna.

Per quanto riguarda i monogatari più importanti, non c'è disgrazia peggiore di non conoscere il Genji monogatari. Leggi bene i manoscritti che ho raccolto per te considerandoli il mio più importante ricordo. Poiché devi conoscere dettagliatamente anche i commentari sui passi più difficili, nella stessa piccola cesta in cui ho messo i manoscritti ho sistemato anche i commentari che avevo affinché tu li possa leggere tutti e comprendere passi del testo poco chiari.

YANASE 1984: 120

La Lettera di Abutsu contiene numerose citazioni di opere della letteratura precedente che includono l'Ise monogatari ("Racconti di Ise", inizio x sec.), raccolte poetiche imperiali o private e aneddoti di ispirazione buddhista. Un posto particolare è però riservato al Genji monogatari al quale Abutsu sembra far riferimento più o meno consapevolmente rievocando a seconda dei casi scene memorabili della storia oppure espressioni trovate qua e là all'interno nel testo. Il capolavoro di Murasaki Shikibu, fornendo come altri monogatari prodotti nell'epoca Heian numerose informazioni riguardo alla vita delle donne della corte, rappresenta una fonte di riferimento indispensabile per scrivere un manuale sull'educazione femminile. Abutsu non si limita solo a riprenderne pedissequamente delle citazioni per fare sfoggio della sua cultura. Anche il suo modo di sentire e di vedere coincide in alcuni casi con quanto è descritto nel Genji monogatari a dimostrazione di quanto fosse naturale e spontaneo per lei ispirarsi a un'opera di cui aveva una conoscenza molto approfondita (Tabuchi 2003: 259-60). 


\section{L'obiettivo finale: conquistare un uomo molto importante}

Il Genji monogatari costituisce una fonte ricca di modelli di donne da emulare, visto che presenta diverse eroine sfortunate, ovvero nobili decadute, come la Dama di Akashi che, nonostante la lunga permanenza in una remota provincia, riesce a conquistare il cuore di un uomo di alto rango come il Principe Genji al quale aveva poi in seguito dato una figlia destinata a diventare imperatrice. Proprio il destino della Dama di Akashi rappresenta per Abutsu un utile esempio di riferimento a cui deve ispirarsi la figlia.

Nell'assurdità del mio folle cuore vorrei che tu diventassi una persona di rango molto elevato e fossi rispettata come madre del sovrano di questo Paese. Sarebbe l'unica consolazione per me in questo mondo effimero. Se poi questo desiderio non si realizzasse, sarà più triste per me brancolare tra le tenebre nella mia vita futura [...] Potrebbe sembrare che io mi prenda gioco di te come un genitore che vuole ad ogni costo farti diventare la consorte del Drago, Sovrano degli Abissi, ${ }^{7}$ nonostante non abbia i mezzi per riuscirci. In realtà, il mio unico, grande desiderio e che tu sia rispettata tra le persone potenti. Ti prego non tradire questa mia volontà.

YANASE 1984: 124-25

Il Drago, Sovrano degli Abissi, è un chiaro riferimento al capitolo Suma del Genji monogatari, in cui si descrive la visione di questo sovrano che appare in sogno a Genji mentre infuria la tempesta poco prima che incontri la Dama di Akashi.

Anche Sua Signoria [Genji] aveva appena chiuso gli occhi, quando accanto a sé vide un essere che egli stesso non poteva definire e che si aggirava come in cerca di qualcuno.-Vi ho chiamato a corte, ma perché non siete venuto?-Si destò, chiedendosi se per caso il Drago, Sovrano degli Abissi, che ama tutto ciò che è bello, non avesse messo gli occhi su

7 Il Drago Sovrano degli Abissi che ricorre nelle leggende giapponesi deriva dal racconto buddhista Sāgara, uno degli otto grandi sovrani Nāga. La figlia, la Principessa Nāga, è colei che nel dodicesimo capitolo del Sūtra del Loto riesce a trasformarsi in uomo e a raggiungere l'illuminazione. 
di lui e questo pensiero lo fece rabbrividire e lo convinse che ormai non poteva più restare in quel luogo.

ORSI 2012: 278

A differenza di altri episodi del Genji monogatari che si aprono con la scoperta di una donna da parte del protagonista, la storia della dama di Akashi inizia con uno sguardo sulla famiglia dell'eroina e le sue aspettative. Il padre della dama era figlio di un ministro che, dopo essere diventato Capitano delle Guardie di Palazzo, aveva ricevuto l'incarico di governatore della provincia di Harima. Quando Genji lo incontra si era ormai ritirato ad Akashi per prendere i voti, ma non aveva ancora abbandonato l'idea di un ritorno agli antichi splendori della sua famiglia nella capitale (Shirane 1987: 74-75). In realtà sarà poi la figlia della Dama di Akashi, Akashi no Kimi, avuta da Genji, a rappresentare di fatto la salvezza per la famiglia, visto che Genji deciderà a un certo punto di portarla nella capitale perché possa avere l'educazione necessaria a diventare una consorte imperiale. La Dama di Akashi rappresenta per Abutsu il modello ideale di donna da imitare non solo perché ha una relazione con un principe imperiale, ma soprattutto perché riesce a stabilire un legame forte e duraturo con lui grazie alla nascita di una figlia. Il punto d'arrivo dell'educazione di una dama non è infatti solo farsi amare da un uomo di rango elevato ma riuscire a dargli dei figli che possano garantire la continuità della famiglia d'origine rafforzandone la posizione. A questo proposito è bene ricordare che quando Abutsu scrive la sua lettera la società e la corte descritte nel Genji monogatari sono ormai molto cambiate, e se nell'epoca Heian solo donne della famiglia Fujiwara potevano ambire a diventare consorte imperiale, con l'affermazione del governo militare e il conseguente sistema dell'imperatore abdicadario, anche una dama di rango non molto elevato, dotata delle virtù necessarie, poteva diventare la madre di principi imperiali, talvolta anche senza un rito maritale che ufficializzasse l'unione (Tabuchi 2003: 262).

Abutsu invita la figlia a leggere con attenzione la storia dei tempi passati per trovare esempi di donne che sono riuscite a diventare consorti imperiali e le fornisce vari consigli utili per riuscire a realizzare questo scopo. Dalle pagine della lettera deduciamo che la candidata ideale deve essere una donna colta, sensibile, raffinata, discreta e umile che più che per la bellezza eccelle per le sue virtù e il suo talento, di cui non fa mai sfoggio in pubblico. Qualora poi avesse la straordinaria fortuna di conquistare l'imperatore sbaragliando anche per poco le altre possibili rivali, non dovrà mai ostentare un'aria di superiorità per non rendersi odiosa e soprattutto dovrà imparare a reprimere in ogni circostanza i suoi sentimenti senza mostrarsi addolorata o risentita nemmeno quando l'interesse del sovrano per lei sembrerà affievolirsi. 
Generalmente è raro attirare l'attenzione dell'imperatore ma anche difficile sfuggirgli. Ad ogni modo, qualora una dama pensi di essere riuscita a conquistarlo almeno un po', non deve mostrarsi tronfia per il risultato conseguito perché ciò la renderebbe odiosa agli occhi degli altri che non farebbero altro che criticarla. Se poi i sentimenti dellimperatore cambiassero e lei si sentisse depressa per questo, poiché sarebbe imbarazzante, non deve parlarne, né darlo a vedere mostrandosi risentita. Anche per quanto riguarda i compiti che le sono stati assegnati a corte, non deve dire che sono fastidiosi e tendere ad accumularne tanti ripetutamente aspettandosi comprensione dagli altri. Non va bene neanche che si mostri orgogliosa della sua bellezza e del suo potere. Chi è incline a comportarsi in questo modo come rugiada svanisce in fretta dalla corte per fare ritorno a casa. E la sua frivolezza diventa subito argomento di pettegolezzi. Anche questo è sicuramente da evitare.

Se poi una dama che non riceve più attenzioni dall'imperatore dicesse: "Come sono depressa! Voglio distrarmi un po'", e decidesse di andare a fare un pellegrinaggio da sola, dando spiegazioni plausibili come: "Per un po' non ci sarà modo di alleviare la mia tristezza", oppure: "In verità ogni tanto vorrei alleggerire il peso dei miei peccati" (Yanase 1984: 127-28), sarebbe sicuramente denigrata. In questo caso se i sentimenti dell'imperatore non sono profondi naturalmente svaniranno senza lasciare alcuna traccia

\section{Inevitabili alternative in caso di fallimento}

Se dopo tanto impegno una dama non riuscisse a raggiungere l'obiettivo prefissato, non ha alternative se non quella di prendere i voti e voltare per sempre le spalle a questo mondo.

Se le cose non vanno come speri, poiché la vita non è eterna, allontanati da questa catena di morte e rinascita e con la nobile intenzione di seguire la via dei bodhisattva, placa le tue passioni, cambia il tuo aspetto e intraprendi il sentiero della verità.

YANASE 1984: 126

Comprendere l'inevitabilità di questa soluzione ci porta a indagare sulla condizione della donna in Giappone nel Medioevo e sul significato che aveva assunto il monachesimo. Nell'epoca Heian esso rappresentava una fase particolare dell'esistenza che iniziava spesso quando le donne, avendo ormai 
adempiuto i doveri di moglie e madre, decidevano con l'avanzare degli anni di dedicarsi alle pratiche ascetiche per ottenere la salvezza nella vita futura. Non mancavano poi i casi in cui questa scelta poteva arrivare anche prima come una forma di divorzio dal marito o semplicemente come un gesto di ribellione rispetto alle imposizioni di una rigida società patriarcale. Dall'epoca Kamakura in poi, con la progressiva affermazione degli ideali confuciani in una società di guerrieri, vivere in ritiro diventa una dimostrazione di fedeltà nei confronti della famiglia di appartenenza. Questo cambiamento della concezione del monachesimo sembra strettamente collegato alla trasformazione dell'istituzione della famiglia che a partire dal XII sec. considera la sposa un membro acquisito nella casa del marito nei confronti del quale doveva mostrare dedizione assoluta per tutta la vita. Stando ai documenti letterari dell'epoca Kamakura, ci si aspettava addirittura che una donna virtuosa prendesse i voti entro i quarantanove giorni dalla eventuale scomparsa del marito dedicando la vita alla preghiera per la salvezza del suo congiunto (Meeks 2010: 1-2).

Per quanto la dimostrazione della fedeltà potesse essere un motivo importante per prendere i voti, pare che le vedove fossero talvolta costrette a farsi monache anche per necessità economiche. Se si considera infatti che il sistema legale vigente non riconosceva a quelle che si risposavano il diritto di ricevere in eredità le proprietà del marito, ${ }^{8}$ possiamo facilmente dedurre che dedicarsi alla vita religiosa, votandosi alla castità, rappresentava l'unica via d'uscita per poter gestire i beni lasciati dal coniuge e stabilire come dovessero essere suddivisi tra i figli (Hosokawa 2006: 58-61).

Lidea di prendere i voti unicamente per manifestare la fedeltà, senza alcun calcolo di tipo economico, sembrerebbe invece più consona a una dama di corte che, non essendo riuscita a stabilire un'unione stabile e duratura con il sovrano presso il quale aveva prestato servizio, preferiva il monachesimo alla possibilità di cercare la protezione di un altro personaggio altolocato. Proprio a questo concetto di fedeltà assoluta al proprio signore, tipico della società samuraica, fa riferimento Abutsu che, senza tralasciare nessun aspetto dell'educazione di una dama dal comportamento impeccabile, indica alla figlia la strada giusta da seguire, cioè prendere i voti per vivere con dignità il suo eventuale fallimento come consorte imperiale e difendersi dalle inevitabili critiche della società circostante. Una opinione analoga è presente anche in altre opere letterarie della stessa epoca, come ad esempio il Towazugatari, nel quale il padre della Dama Nijō sul letto di morte spiega alla figlia perché in certi casi è più rispettabile diventare monaca piuttosto che cambiare signore.

8 Ci riferiamo in particolare all'articolo 24 del Goseibai shikimoku ("Codice di Giustizia"). 
Se servi il tuo sovrano in modo che nessuno abbia qualcosa da ridire, non devi fare altro che continuare a svolgere i tuoi compiti con attenzione e solerzia. Se invece, come capita in questo mondo in cui le cose non vanno sempre come si desidera, ti sentissi criticata dal tuo signore e dagli altri e non avessi la forza necessaria per affrontare la situazione, intraprendi la strada del Buddha. Contribuirai così alla tua salvezza e mostrerai riconoscenza ai tuoi genitori pregando affinché possiamo rinascere tutti insieme nella nostra vita futura. Se invece, pensando di essere stata abbandonata e di non avere nessuno a cui fare affidamento, decidessi di metterti al servizio di un qualsiasi altro signore, allora, anche dopo la mia morte, penserei di essere stato disonorato.

KUBOTA 2008: 165

Fallire significava tradire le aspettative della propria famiglia e sentirsi responsabili delle inevitabili conseguenze, compreso il disonore. Il padre della Dama Nijō chiaramente spera di avere dei vantaggi politici dalla relazione della figlia con l'imperatore GoFukakusa, ma la figlia, pur mostrandosi all'inizio capace e ambiziosa, a poco a poco perde l'occasione di diventare consorte imperiale ed è costretta ad abbandonare la corte per la sua incapacità di garantire un erede. ${ }^{9}$

La possibilità di assicurarsi la protezione duratura di un uomo influente mettendo al mondo un figlio che poteva in futuro acquisire una posizione politica importante assicurava nell'epoca Kamakura una notevole mobilità sociale e rappresentava per molte donne e le loro famiglie appartenenti ai medi ranghi della nobiltà un sogno da inseguire investendo con determinazione tutte le proprie forze. La vita della stessa Abutsu, autrice della lettera, è una testimonianza della realizzazione di questo ambizioso progetto grazie al matrimonio con Fujiwara no Tameie e la nascita dei figli avuti da lui. Da vedova, pur prendendo definitivamente i voti, continuerà a lavorare e a combattere per la famiglia come donna istruita, consapevole del proprio valore e dei diritti che le spettavano. Nella sua esperienza di vita si incrociano naturalmente vari tipi di problemi che hanno caratterizzato la storia delle donne non solo in Giappone, ma anche in altri paesi del mondo: ricerca di risorse finanziarie, riconoscimento di diritti giuridici, necessità di essere iscritte non solo nella vita riproduttiva ma anche in quella intellettuale. Tutti temi di cui gli studiosi da sempre discutono nel tentativo di ricostruire una parte della storia e assegnare un posto più adeguato a chi ancora non sembra averlo. Si tratta di

9 Riguardo alla storia della Dama Nijō e alle motivazioni che la costringono a prendere i voti si vedano Sarra (2001) e Kimura (2007). 
una ricerca impegnata e coinvolgente che implica la considerazione dell'inserimento delle donne nell'insieme dei rapporti sociali e nel tempo storico in cui hanno vissuto.

\section{Riferimenti bibliografici}

Fukuda, H. (1969) Abutsu ni, in S. Hisamatsu (a c.), Nihon joryū bungaku shi, 388-410. Tōkyō.

Hosokawa, R. (2006) Nihonshi no josei shin no jitsuryoku—sōzoku to isan. Rekishi tokuhon 51/11, 54-61.

Ishida, J. (1996) Makura no sōshi (Kadokawa bunko Sophia 32), 2 voll. Tōkyō.

Iwasa, M. (1999) Kyūtei joryūbungaku dokkai kō. Chūseihen. Tōkyō.

Kimura, S. (2007) Regenerating Narratives. The Confessions of Lady Nijō as a Story for Women Salvation. Review of Japanese Culture and Society 19, 87-102.

Kubota, J. (2008) Towazugatari, in Y. Kikuchi et al. (a c.), Tosa nikki, Kagerō nikki, Towazugatari (Nihon no koten o yomu 7), 144-285, Tōkyō.

Laffin, C. (2013) Rewriting Medieval Japanese Women. Politics, Personality, and Literary Production in the Life of Nun Abutsu. Honolulu.

Matsumoto, Y. (1983) Chūsei joryū nikki bungaku no kenkyū. Tōkyō.

Meeks, L. (2010) Buddhist Renunciation and the Female Life Cycle. Understanding Nunhood in Heian and Kamakura Japan. Harvard Journal of Asiatic Studies 70/1, $1-59$.

Nagasaki, K., O. Hamanaka (1996) Kōdō surujosei. Abutsu ni (Nihon no sakka 22). Tōkyō.

Negri, C. a c. (2000) La principessa di Sumiyoshi. Sumiyoshi monogatari. Venezia.

Negri, C. a c. (2008) Diario di Izumi Shikibu. Izumi Shikibu nikki. Venezia.

Orsi, M.T. a c. (2012) La storia di Genji. Torino.

Sarra, E. (2001) Towazugatari. Unruly Tales from a Dutiful Daughter, in R.L. Copeland, E. Ramirez-Christensen (eds.), The Father-Daughter Plot. Japanese Literary Women and the Law of the Father, 89-114. Honolulu.

Shirane, H. (1987) The Bridge of Dreams. A Poetics of The Tale of Genji. Stanford.

Suzuki, E. (1936) Jokun. Menoto no fumi. Tōkyō.

Tabuchi K., (2003) Abutsu ni no Genji monogatari kyōju. Menoto no fumi o chūshin ni, in K. Suzuki, T. Ito (a c.), Genji monogatari kanshō kiso chishiki: 28, 254-68. Tōkyō.

Tabuchi K. (2009a) Izayoi nikki hakubyōtansai eiri shahon. Abutsu no fumi. Tōkyō.

Tabuchi K. (2009b) Abutsu ni. Tōkyō.

Takagi, S. (2010) Abutsu no fumi ron. Kisaki gane no kokorogamae o megutte. Kokugo to kokubungaku $87 / 8,32-47$.

Wakita, H. (1984) Marriage and Property in Premodern Japan from Perspective of Women's History. The Society for Japanese Studies 10/1, 73-99.

Yanase, K. ed. (1984) Abutsu ni zenshū zōhōban. Tōkyō. 